\title{
Bombarder pour convaincre ? Puissance aérienne, rationalité limitée et diplomatie coercitive au Kosovo
}

\author{
Pascal Vennesson
}

\section{(2) OpenEdition \\ Journals}

\section{Édition électronique}

URL : http://journals.openedition.org/conflits/395

DOI : 10.4000/conflits.395

ISSN : $1777-5345$

Éditeur :

CCLS - Centre d'études sur les conflits lilberté et sécurité, L'Harmattan

Édition imprimée

Date de publication : 1 mars 2000

ISBN : 2-7384-9385-8

ISSN : $1157-996 \mathrm{X}$

\section{Référence électronique}

Pascal Vennesson, «Bombarder pour convaincre? Puissance aérienne, rationalité limitée et diplomatie coercitive au Kosovo », Cultures \& Conflits [En ligne], 37 | printemps 2000, mis en ligne le 20 mars 2006, consulté le 30 mars 2021. URL : http://journals.openedition.org/conflits/395; DOI : https://doi.org/10.4000/conflits.395

Ce document a été généré automatiquement le 30 mars 2021.

Creative Commons License 


\title{
Bombarder pour convaincre? Puissance aérienne, rationalité limitée et diplomatie coercitive au Kosovo
}

\author{
Pascal Vennesson
}

Usages et utilité de la force armée au début du XXIème siècle¹. Le 24 mars 1999, les forces aériennes des pays membres de l'Organisation du traité de l'Atlantique nord et au premier chef, celles des Etats-Unis, débutaient l'opération «Force alliée », une campagne aérienne majeure contre la République Fédérale de Yougoslavie. Le 10 juin 1999, après 79 jours de bombardements, à la surprise et au soulagement des décideurs et des analystes occidentaux, les dirigeants serbes acceptaient les exigences des leaders politiques des pays membres de l'OTAN. Cette crise internationale comporte de nombreux aspects et elle a, dores et déjà, suscité une explosion de commentaires et d'analyses sur l'histoire des Balkans, le nationalisme et ses conséquences, l'armée de libération du Kosovo, les interventions militaires et leur justification du point de vue du droit international, les réfugiés, la conduite de négociations diplomatiques, le devenir de l'OTAN, les relations transatlantiques, la défense européenne, l'action du tribunal pénal international, la sociologie politique des intellectuels, etc ${ }^{2}$. Comme il est courant dans tous les conflits, les apparences et les premiers constats au cours des hostilités, surtout à partir de sources de presse, ont été trompeurs et plusieurs informations sont venues, depuis juin 1999, apporter une première série de corrections aux perceptions initiales. Au fur et à mesure de la diffusion d'informations nouvelles, notre interprétation de la crise se transformera encore, et sans doute considérablement. L'objectif du présent article est de situer la crise du Kosovo par rapport aux principales conceptions de la diplomatie coercitive et de la puissance aérienne et d'avancer une interprétation de l'utilisation de l'arme aérienne ${ }^{3}$. Quels étaient les objectifs de la campagne aérienne? Comment a-t-elle été conçue et mise en œuvre? Quels facteurs politiques et militaires ont affecté sa planification et son exécution? Quel a été l'impact de la puissance aérienne sur la décision des dirigeants serbes de mettre un terme à 
leurs actions? Les premiers enseignements déjà tirés n'épuisent pas, loin s'en faut, les réflexions sur la crise du Kosovo et ses conséquences sur la compréhension des usages de la force armée aujourd'hui ${ }^{4}$. La première étape est de préciser la nature même de l'emploi de la force armée et ses relations au politique au cours de la crise. La catégorie "guerre ", utilisée à l'envie par la plupart des observateurs, et presque exclusive dans les lectures médiatiques de l'épreuve de force, s'est révélée trop générale pour identifier et clarifier les logiques de l'usage de la force. Après coup, les praticiens ont aussi rationalisé leur action en utilisant des termes différents de ceux qu'ils avaient utilisés au cours de la crise. Cet effort de définition est d'autant plus indispensable qu'une tendance de la réflexion depuis la fin de la Guerre Froide tend à sous-estimer la pertinence de la puissance et de la force armée, à considérer leur emploi obsolète et à proposer un important élargissement de la notion de sécurité, au-delà des aspects proprement militaires. L'inconvénient de cette orientation a été de renforcer l'indifférence des spécialistes de sciences sociales pour les articulations du militaire et du politique qui devraient pourtant constituer l'un de leurs objets de recherche naturels. Au bout du compte, les spécialistes de sciences sociales comme les citoyens, se trouvent dépourvus des instruments intellectuels, des grilles de lectures, des références théoriques et empiriques leur permettant de décoder ce type d'événements. Une deuxième étape permettra de présenter les différentes conceptions de la puissance aérienne et de sa mise en œuvre, ainsi que d'analyser leur apport et leurs limites au cours de la crise. Dans un troisième temps, enfin, on avancera une autre lecture des usages de la puissance aérienne dans la diplomatie coercitive. Nombre de débats sur la puissance aérienne au cours de la crise du Kosovo ont tourné à un face à face stérile entre les partisans du tout aérien et les sceptiques. La plupart des interprétations concurrentes de la crise ont fini, à force de remettre en cause (avec pertinence d'ailleurs) les lectures naïvement excessives de la contribution de la puissance aérienne, par créer une sorte de consensus minorant l'impact de la puissance aérienne sur l'issue de la crise, négligeant certaines de ses contributions effectives autant que les processus qui sous-tendent sa mise en œuvre. La principale thèse défendue ici est que, même si ces réserves sont dans l'ensemble pertinentes (elles dénoncent souvent des positions si excessives que leur mérite est cependant moins grand qu'il n'y paraît), elles sous-estiment l'impact politico-diplomatique de la puissance aérienne. Pour saisir ce rôle, il convient d'adopter le modèle de rationalité limitée d'Herbert Simon et les conceptions de l'action publique qui en découlent. La plupart des conceptions de la stratégie aérienne sont, en effet, fondées, implicitement au moins, sur un modèle de rationalité fondé sur l'optimisation (ou maximisation) qui ne peut véritablement rendre compte des logiques de l'utilisation de la puissance aérienne en général, et au cours de la crise du Kosovo en particulier. En adaptant et en proposant un début d'application du modèle de « rationalité limitée » ou " procédurale » mis au point par Herbert Simon, on peut critiquer et enrichir certaines conceptions stratégiques et mieux saisir les logiques de l'emploi de la force armée aujourd'hui. Puissance aérienne et diplomatie coercitive : définitions Qu'est-ce que la puissance aérienne? L'analyse ici privilégiée porte sur la dimension militaire de la crise, sur les considérations militaires - précisément ici, celles qui sont liées à la puissance aérienne - qui sous-tendent les logiques de la coercition, ainsi que sur la relation entre ces aspects et les bénéfices politiques espérés 5 . Comment les décideurs qui envisagent l'utilisation de la force sontils influencés par leur estimation des conséquences de ce choix sur l'issue de la crise? Comme l'affrontement du Kosovo a été marqué par l'action diplomatique et militaire 
des Etats-Unis, il est indispensable de tenir compte de cette caractéristique majeure. Les conceptions de la puissance aérienne et de ses usages ici évoqués sont largement (mais pas exclusivement) celles qu'adoptent, ou rejettent, les officiers de l'armée de l'air et les décideurs politiques américains ${ }^{6}$. Il s'agit également de l'une des limites de notre interprétation: nous aurons tendance à reporter à un autre travail les conceptions, perceptions et actions des pays européens, et notamment de la France, du Royaume-Uni et de l'Allemagne. Par exemple, le président Jacques Chirac a effectivement mis son veto à certaines frappes aériennes. Ce point est important pour comprendre la politique de la France, certaines actions des Etats-Unis (notamment les frappes américaines conduites en dehors de l'OTAN) et, plus généralement, les relations entre les Etats-Unis et l'Europe. Au bout du compte cependant, ce sont des platesformes américaines qui ont délivré plus de $80 \%$ des munitions utilisées 7 . Au sens large, l'expression "puissance aérienne » désigne l'utilisation de l'air et de l'espace à des fins militaires. La puissance aérienne est un type particulier de puissance militaire générée par des plates-formes en mesure d'effectuer un vol soutenu, manœuvrable et motorisés. Cette définition large est analytiquement la plus utile. Elle nous permet d'abord d'éviter de reprendre spontanément les catégories usuelles qui proviennent de la division du travail entre les armées. La puissance aérienne ne signifie pas exclusivement l'armée de l'air. Aucune armée n'exerce un monopole parfait sur la puissance aérienne. Certains satellites, des avions de reconnaissance (en France, l'aviation légère de l'armée de terre), la plupart des hélicoptères et même certains avions (l'aéronavale) relèvent d'autres armées. Si d'épineuses questions organisationnelles se posent ici sur la répartition des tâches entre les organisations concernées, et parfois en leur sein, l'essentiel est qu'une analyse complète de la puissance aérienne ne saurait se confondre avec, ou se limiter à l'étude de l'action d'une armée. Cette définition nous épargne également le réductionnisme technologique. La puissance aérienne ne se résume pas aujourd'hui aux avions et elle sera même peut-être tendanciellement de moins en moins caractérisée par les avions pilotés. Il faut y inclure, en tous cas, les hélicoptères, dont certains disposent aujourd'hui d'une puissance de feu supérieure à nombre de chasseurs-bombardiers du passé, mais également des missiles à longue portée (100 km et davantage), ou des drones, de petits appareils télécommandés qui collectent des renseignements et mènent des actions de guerre électronique. Enfin, même si nous allons nous concentrer ici sur des usages coercitifs de l'air, cette définition large nous rappelle opportunément que la puissance aérienne n'est pas exclusivement létale. Le transport aérien militaire permet de déplacer rapidement des forces et de les approvisionner, les systèmes de surveillance du champ de bataille utilisent des radars aériens. Parmi les missions aériennes directement létales on peut distinguer (même si ces distinctions sont partiellement arbitraires), outre la supériorité aérienne (obtenue par le combat aérien), l'appui au sol et le bombardement stratégique ${ }^{9}$. Au cours de l'opération «Force alliée ", les pays membres de l'OTAN ont déployé un dispositif aérien considérable qui comprenait tous les aspects de la puissance aérienne: défense aérienne, assaut conventionnel, communications et renseignement, guerre électronique, protection visà-vis des menaces antiaériennes, etc ${ }^{10}$. Pour en rester à un contexte récent, depuis la fin de la Guerre Froide, la puissance aérienne a connu une utilisation intensive, notamment par les Etats-Unis. Outre la guerre du Golfe et la guerre en Bosnie, la puissance aérienne a été mise en œuvre en Somalie, des frappes aériennes ont été utilisées par les Etats-Unis en août 1998 au Soudan (contre une entreprise 
pharmaceutique soupçonnée de dissimuler la fabrication d'armes chimiques) et simultanément en Afghanistan (contre les camps d'entraînement de groupes dirigés par Osama ben Laden suspectés de terrorisme), contre l'Irak sous la forme d'une surveillance de zones aériennes interdites aux Irakiens au nord et au sud du pays, puis de frappes presque ininterrompues sur le potentiel militaire irakien à partir de décembre 1998 et au cours de la crise Bosniaque. Même s'il n'est pas inédit, y compris dans des guerres limitées, voire dans des opérations de contrôle des colonies, par exemple, l'usage de l'arme aérienne s'est banalisé depuis la fin de la Guerre Froide. L'évaluation des effets de la puissance aérienne - et plus généralement d'ailleurs des opérations militaires - est une entreprise, pratique autant que théorique, semée d'embûches. Cet effort fait voler en éclat les divisions du travail communément acceptées entre les praticiens, forcément athéoriques, et les théoriciens, par définition coupés des réalités. En fait, les praticiens construisent des hypothèses, identifient des variables dépendantes et indépendantes, se livrent à des enquêtes empiriques pour mesurer l'influence de ces variables, mènent une réflexion sur l'éventuelle généralisation des conclusions auxquelles ils sont parvenus sur un cas précis. Tout cela ressemble beaucoup au pain quotidien des sciences sociales. De plus, l'évaluation des forces militaires de l'adversaire et de son économie, de l'impact des opérations en cours constituent des entreprises complexes, souvent marquées par la dispersion des informations, le cloisonnement dans la diffusion du renseignement et les conflits entre intérêts organisationnels. Il faut marquer quelques unes des limites de cette réflexion. La principale difficulté, contrairement aux impressions complaisamment entretenues sur la guerre vécue en direct grâce à une couverture médiatique censée assurer une remarquable transparence, provient du manque de données fiables sur le déroulement du conflit, sur les préférences et les perceptions des acteurs et notamment des décideurs serbes, dont Slobodan Milosevic lui-même, ainsi que, pour les origines de la crise, sur les représentations et les stratégies des dirigeants de l'armée de libération du Kosovo. Par ailleurs, l'évaluation précise des effets de différents facteurs, comme l'utilisation de l'arme aérienne, sur le déroulement des opérations et l'aboutissement de la crise, demeure problématique. Toute analyse systématique menée sur ce cas devrait examiner également plusieurs scénarios "contrefactuels» du type, que ce serait-il passé si... ? De plus, comme souvent dans les relations internationales, les décideurs politiques et militaires ont intérêt à présenter de manière délibérément sélective, voire trompeuse, les événements en cours. Par exemple, au cours de la crise puis de la guerre du Golfe, le président américain George Bush et son équipe ont répété à l'envie, contrairement à l'image dominante de ce qui s'était produit au cours de la guerre du Vietnam, qu'ils laissaient les militaires libres d'agir à leur guise dans la conduite des opérations et ne se livreraient pas à un "micro-management» des opérations. Cette absence d'interférence des décideurs civils permettrait aux militaires de privilégier les considérations opérationnelles et de parvenir efficacement à la victoire. En fait, à l'inverse de leurs protestations publiques, les décideurs politiques n'ont cessé d'intervenir dans la conduite des opérations (par exemple dans la sélection des cibles des bombardements). Le Secrétaire à la défense Richard Cheney, qui s'était rapidement formé aux aspects fondamentaux de l'art opératif, a exercé un strict contrôle des opérations militaires ${ }^{11}$. Les décalages entre les représentations et analyses les plus proches de l'événement et les analyses et interprétations futures sont donc monnaie courante. Les analyses des conflits et les leçons que les acteurs en tirent, ou devraient en tirer, se transforment aussi, parfois considérablement au fil du temps, 
comme c'est encore le cas aujourd'hui pour la guerre du Golfe ${ }^{12}$. Si l'on se concentre sur le problème plus spécifique de l'évaluation des effets des campagnes aériennes, les limites et les difficultés sont aussi marquées. Dans les premiers temps de la campagne aérienne menée par l'OTAN, les idées reçues (le bombardement stratégique n'a pas conduit l'Allemagne à capituler, par exemple) ont supplanté l'analyse rigoureuse des connaissances existantes. En 1944, le choix entre les différentes options disponibles pour mettre en œuvre efficacement la puissance aérienne a été complexe et influencé par la rivalité des conceptions, des organisations et des acteurs ${ }^{13}$. L'effet des bombardements contre l'Allemagne nazie au cours de la Seconde Guerre Mondiale a été explicitement évoqué au cours de la crise, notamment par l'économiste John Kenneth Galbraith qui avait dirigé le «U. S. Strategic Bombing Survey» en 1945. Il concluait qu'au cours du conflit mondial le bombardement avait été, au total, inefficace, mais son avis est loin d'être unanimement partagé ${ }^{14}$. Les travaux disponibles, aussi bien les enquêtes réalisées à l'époque que les travaux historiques effectués depuis, révèlent un panorama plus contrasté. Les bombardements ont bien eu un impact, parfois significatif, sur les Etats qui en faisaient les frais. Des enquêtes approfondies sur l'approvisionnement en charbon en Allemagne en 1944-45, par exemple, ont montré que la désorganisation du système ferroviaire par les bombardements anglo-américains avait produit des effets dévastateurs sur l'économie de guerre allemande ${ }^{15}$. L'influence des bombardements américains sur le Japon, y compris l'influence des bombes atomiques d'Hiroshima et de Nagasaki, sur la décision du Japon de capituler sont également discutées, mais bien plus riches que les conclusions qui tendent à exagérer ou à négliger ces conséquences ${ }^{16}$. Qu'est-ce que la diplomatie coercitive ? Les notions de "guerre ", et parfois de "dissuasion", constamment utilisées dans les traitements médiatiques de la crise du Kosovo, accompagnées par des visions étroites des usages de la force armée, ont conduit à des perceptions confuses des événements. En fait, l'utilisation de la puissance aérienne dans la crise du Kosovo relève de la diplomatie coercitive, c'est-à-dire une menace et/ou un emploi volontairement limité et graduel de la puissance militaire afin de persuader un adversaire de mettre un terme à une action en cours, de revenir au statu quo ante, ou de l'obliger à une action qu'il juge indésirable ${ }^{17}$. Il ne s'agit donc pas de gagner une guerre au sens militaire classique. Principalement préoccupés par la dissuasion nucléaire, et soucieux de définir et de légitimer la position de la France en ce domaine, les spécialistes français de stratégie connaissent mal et interprètent de manière réductrice cette notion de " compellence ». S'il précise clairement la distinction entre dissuasion et persuasion, Lucien Poirier semble accepter l'idée selon laquelle la diplomatie coercitive exclut l'emploi effectif de la force, ce serait à ses yeux son point commun avec la dissuasion. Or, Thomas Schelling prend soin de préciser à de nombreuses reprises que certains usages matériels de la force sont partie intégrante de la coercition ${ }^{18}$. Lorsqu'il évoque spécifiquement la "stratégie de persuasion (compellence) », Hervé Coutau-Bégarie résume l'argument de Schelling au seul fait de brandir la menace de l'arme nucléaire au cours d'une crise pour contraindre l'adversaire à agir en un sens déterminé ${ }^{19}$. Même si Schelling évoque effectivement ce point important, et les difficultés qu'il comporte, il ne s'agit que d'un aspect d'une réflexion qui porte davantage sur l'utilisation de forces conventionnelles dans les relations internationales. Les termes et expressions " coercition ", « coercition stratégique ", "diplomatie coercitive ", "compellence ", "stratégie de persuasion ", "stratégie de chantage » (blackmail strategy), "stratégie de contrainte », " diplomatie de la canonnière " sont souvent utilisés de manière interchangeable pour désigner 
cette diplomatie de la violence qui se sert de la force armée pour « exploiter les peurs et les désirs de l'ennemi $»^{20}$. S'il n'existe pas de consensus absolu sur les termes permettant de désigner cette réalité, que nous résumons ici sous l'expression "diplomatie coercitive", il existe en revanche, avec quelques nuances, un assez large accord sur les réalités qu'elle recouvre. C'est à la fin des années 1950 et au début des années 1960, dans le cadre de plusieurs recherches menées à la RAND Corporation notamment celles de Daniel Ellsberg d'abord et d'Alexander George - que le verbe " to compel » (contraindre, astreindre, forcer, obliger, pousser à) et le terme " compellence » sont utilisés pour la première fois et désignent la menace du recours à la force dans les relations internationales. Ces travaux ne seront publiés qu'à la fin des années 1960 et c'est Thomas Schelling qui va, dans Arms and Influence publié en 1966, fournir la définition la plus complète de la notion et présenter l'une de ses utilisations les plus fécondes ${ }^{21}$. Schelling a proposé le mot « compellence » non sans hésitations et scrupules. Le terme n'est pas toujours accepté en anglais sous sa forme adverbiale et n'est pas aisément traduisible en français. Le terme " persuasion » est proche et saisit bien l'idée d'amener quelqu'un à croire, à faire, à vouloir quelque chose. Mais il a l'inconvénient de gommer la contrainte physique. On dira, par exemple, qu'il faut recourir à la persuasion plutôt qu'à la force, alors même que la "compellence " comporte la menace et certains usages de la force pour obliger l'adversaire à agir. La diplomatie coercitive est distincte de la dissuasion, comme de la stratégie militaire conventionnelle ${ }^{22}$. Dissuader c'est faire en sorte que l'adversaire s'abstienne d'agir car ses chances de succès sont trop aléatoires et/ou d'un coût disproportionné aux gains escomptés. Il s'agit d'une stratégie de non-emploi des forces et ce trait est encore plus marqué avec la dissuasion nucléaire. Lorsque l'adversaire passe outre cette menace de punition et lance son action militaire, la dissuasion a échoué. La diplomatie coercitive, en revanche, implique que l'adversaire modifie son comportement d'une manière ou d'une autre. L'acteur qui s'engage dans une opération de diplomatie coercitive ne se contente pas d'attendre, il doit agir pour modifier le statu quo. De plus, la diplomatie coercitive est indéterminée sur le plan des moyens utilisés et elle peut inclure le recours à la force. Schelling souligne que la menace qui contraint à agir, au contraire de celle qui dissuade, implique souvent, mais pas toujours, que la punition soit administrée jusqu'à ce que l'autre agisse, et pas seulement s'il agit. La menace de destructions est fondée sur des destructions réelles. La diplomatie coercitive ne se ramène pas non plus à la stratégie militaire conventionnelle. Schelling contraste la « force brute » et la coercition. Dans le cas de la coercition, la force armée ne sert pas à occuper et tenir un territoire, ou à accomplir une manœuvre offensive visant par la combinaison du feu et du mouvement à détruire un ennemi localisé ou à le chasser des zones qu'il occupe en lui infligeant le plus de pertes possibles. Lorsqu'un pays dispose d'une force militaire suffisante - ou peut utiliser une force militaire de grande ampleur avec peu de restrictions - il n'existe pas véritablement de besoin de marchandage. La reddition sans condition peut même être un cas ultime de ce type de situation. Dans le cadre de la diplomatie coercitive, l'emploi de la force est conçu et réalisé sous la forme d'un marchandage et d'un chantage. Il doit y avoir un intérêt commun entre les adversaires pour dégager un terrain d'entente. La force est utilisée pour faire souffrir, pour détruire de la valeur. Pour Schelling, la capacité à infliger des souffrances peut conduire certains acteurs à vouloir les éviter. Il s'agit d'influencer leur comportement, de contraindre leur décision ou leur choix. « Le pouvoir de faire mal est un pouvoir de marchandage. L'exploiter relève de la diplomatie, une diplomatie vicieuse (vicious), 
mais de la diplomatie tout de même $»^{23}$. La force armée se veut ici maitrisée, contrôlée, voire tenue en réserve. La menace de dommages, ou la perspective de dommages encore plus importants, peut conduire un acteur à faire machine arrière. Ce n'est pas la douleur ou les dommages eux-mêmes qui comptent, mais leur impact sur le comportement de l'adversaire. Au total, la "diplomatie coercitive », ou « diplomatie de la violence ", cherche à persuader un adversaire par la menace de la violence, mais également par un usage limité, graduel et réversible de celle-ci. Dans le cas de la coercition (au contraire de la dissuasion), la menace peut s'actualiser pour parvenir au but recherché. L'épreuve de force peut être effective. Ce type d'usage de la force, s'il a pris une tournure particulière dans un système international marqué par les armes nucléaires et s'il a été théorisé de manière plus systématique au début des années 1960, a emprunté différentes formes dans l'histoire des relations internationales, comme les ultimatums, les sanctions économiques et la diplomatie de la canonnière ${ }^{24}$. La puissance aérienne, flexible, facile à interrompre pour laisser à l'adversaire la possibilité d'accepter les exigences de son opposant, a joué depuis les années 1960 un rôle important dans la diplomatie coercitive. Si au cours de la guerre du Vietnam, l'utilisation de la puissance aérienne a été critiquée, la fin de la Guerre Froide et peutêtre plus encore les suites de la guerre du Golfe, ont marqué le retour de la coercition aérienne ${ }^{25}$. Après la guerre du Golfe, pour contraindre Saddam Hussein et les dirigeants irakiens à accepter les inspections de l'ONU, les leaders politiques et militaires américains ont utilisé la stratégie de coercition à l'encontre de Saddam Hussein et des dirigeants irakiens ${ }^{26}$. Les indices abondent indiquant que la crise du Kosovo a été marquée par les logiques de la diplomatie coercitive abondent. Celle-ci a entraîné les résistances, les tensions et les frustrations qu'elle avait déjà suscitées dans le passé. Par exemple, le général Clark a expliqué : « A la base, les sources politiques de l'OTAN n'ont jamais été satisfaites avec une opération aérienne par phase, parce qu'elles voulaient quelque chose de plus limité, de plus diplomatique (en français dans le texte) $»^{27}$. Certains observateurs ont évoqué la guerre limitée, l'escalade graduée et, en tous cas, la remise en cause, au moins partielle, de la « doctrine Weinberger-Powell». En novembre 1984, le Secrétaire à la défense Caspar Weinberger prononçait un discours intitulé : «les usages de la puissance militaire». A la suite de l'attentat perpétré contre la caserne des Marines en octobre 1983 à Beyrouth et de l'échec de la mission de maintien de la paix, et au moment où les principaux chefs de l'armée américaine ayant vécu la guerre du Vietnam parviennent aux postes de responsabilité, les décideurs américains ont précisé plusieurs critères devant gouverner l'usage de la force. Ces critères insistaient sur le caractère entier des opérations militaires et s'écartaient de toute idée de coercition. Une fois que l'on admet que la campagne aérienne menée de mars à juin 1999 était une action de diplomatie coercitive une série de critiques deviennent sans objet. Ce constat ne signifie pas, au contraire, que ce type d'opération diplomaticomilitaire ne comporte pas d'importantes limites, on y reviendra. Le caractère en partie public de la planification des frappes, qui conduit à sacrifier partiellement l'effet de surprise n'est pas une déficience, ou une limite, mais l'une des composantes de la diplomatie coercitive. Il est indispensable, en effet, d'adresser des signaux, d'envoyer des messages et non de conduire une guerre conventionnelle. L'opération «Force alliée » n'était pas non plus un "ersatz de guerre » qui aurait eu pour objectif de détruire la Serbie, mais un usage particulier de la force armée. Il n'y a pas eu sur ce plan de dissimulation. En mars, aucun des dirigeants politiques et militaires des pays membres de l'OTAN ne voulait livrer une guerre terrestre aux troupes de la RFY. Ce 
sont les frappes aériennes qui ont progressivement conduit à une transformation partielle de ce refus initial. Les commentateurs qui ont critiqué l'imposition d'une « logique de guerre » dans la crise, et l'application aveugle de la force, manquent l'un des aspects essentiels de ce type de recours à la force : l'étroit contrôle politique et diplomatique sur les actions militaires avec, par exemple, l'approbation systématique des cibles et des frappes par les autorités politiques. On devrait évoquer en fait une forte "politisation» de l'usage de la force qui limite (ou s'efforce de limiter) l'autonomie relative des militaires. La force est disciplinée, au service de la politique. Les logiques de la diplomatie coercitive peuvent d'ailleurs comporter des contradictions entre les objectifs politiques et militaires et des conflits entre leaders politiques et militaires. La puissance aérienne au Kosovo : théories et pratiques A quoi sert la puissance aérienne ?28. Contre quels objectifs et à quelles fins? Quelles sont les utilisations de la puissance aérienne qui permettent, en théorie comme en pratique, d'affecter les préférences de l'adversaire? Quelles ont été leurs influences et leurs conséquences, telles qu'on peut les évaluer au moins grossièrement aujourd'hui, au cours de la crise du Kosovo? On peut distinguer cinq types d'emplois des forces, partiellement concurrents et partiellement complémentaires, qui visent différents centres de gravité et reposent donc sur des conceptions différentes (et partiellement divergentes) de la nature même de l'adversaire : la dissuasion, la destruction du centre de gravité politique, économique et social du pays visé, la destruction de ses forces militaires et paramilitaires, l'appui au sol et enfin l'invasion terrestre. Les quatre premiers reposent, à des degrés divers, sur la puissance aérienne. Le dernier est une stratégie militaire conventionnelle qui comporte une invasion du Kosovo. Elle inclut également une action aérienne pour assurer la supériorité aérienne et l'appui des forces au sol, mais il s'agit classiquement d'emporter la décision sur le champ de bataille. Même si l'ensemble de ces facteurs a exercé une influence au cours de la crise du Kosovo, il reste essentiel de les distinguer et de s'interroger sur les modalités de leur combinaison. C'est à cette condition que l'on peut évaluer rigoureusement les différentes options possibles. Du point de vue de la pratique, en effet, les ressources et le temps disponibles sont toujours limités. Il est donc crucial d'allouer au mieux des ressources rares. Ces distinctions sont également essentielles car elles ont provoqué au cours des opérations des divisions et de vifs désaccords entre décideurs politiques et militaires, aussi bien qu'entre les chefs militaires eux-mêmes et particulièrement entre le général Wesley K. Clark (armée de terre), commandant suprême des forces alliées en Europe - Supreme Allied Commander, Europe (SACEUR) et le général Michael Short, son subordonné de rang le plus élevé pour l'armée de l'air. Au cours des premières semaines du conflit, le problème de la répartition des appareils entre le Kosovo proprement dit et le reste de la RFY a, par exemple, constitué une source constante de désaccords. Puissance aérienne et dissuasion Le premier usage de la puissance aérienne est d'ordre dissuasif. En un sens général, dissuader signifie persuader un adversaire de ne pas initier une action spécifique en lui montrant que les bénéfices qu'il escompte tirer de son action ne justifient ni les coûts, ni les risques probables qu'il encourt. L'attaquant se livre à une évaluation des coûts probables. Ces coûts dépendent en grande partie de la durée nécessaire pour atteindre les objectifs recherchés. Plus l'affrontement conventionnel est long, plus les coûts sont élevés. La question qui se pose est donc: est-il possible d'atteindre ces objectifs rapidement? Quels sont les facteurs qui conduisent les décideurs serbes à penser qu'ils peuvent atteindre rapidement leurs objectifs sur le champ de bataille? La réponse potentielle des pays de 
l'OTAN est-elle suffisamment grave, fait-elle suffisamment peur, pour dissuader l'attaque ? Si la réponse à cette question est positive, la dissuasion sera assurée, dans le cas contraire elle n'aura pas lieu. La menace de l'utilisation de la puissance aérienne à l'encontre des acteurs qui n'accepteraient pas les négociations a été régulièrement brandie à propos du Kosovo. A la fin février 1998, les forces militaires et paramilitaires de la RFY entament une offensive contre l'armée de libération du Kosovo (UCK). Entre la fin juillet et la fin septembre, plus de 250000 Kosovars étaient expulsés de leurs villages. A la suite de plusieurs mises en garde, d'un début de planification, de contacts informels entre les responsables militaires au sein de l'OTAN et d'un avertissement adopté le 22 septembre par le groupe de contact sur l'ex-Yougoslavie, le 25 septembre les pays membres de l'OTAN décidaient officiellement d'autoriser le commandant suprême des forces alliées en Europe à demander aux pays membres les forces qu'ils seraient prêts à fournir dans le cas d'une action militaire ${ }^{29}$. Il s'agissait, aux yeux des dirigeants de l'alliance, d'un signal fondé sur une planification opérationnelle. Le 28 septembre, Mirko Marjanovic, le premier ministre de la RFY annonçait que les « activités anti-terroristes » avaient pris fin au Kosovo. De plus, dans le cas de la Bosnie, un précédent apparent pour les dirigeants politiques et militaires des pays membres de l'OTAN pouvait donner une crédibilité à la menace. Ce précédent n'est qu'apparent car ce n'était pas la puissance aérienne seule, mais le succès de l'offensive au sol des troupes des Croates et des musulmans de Bosnie qui avaient contraint les décideurs serbes à retirer leurs forces des alentours de Sarajevo ${ }^{30}$. Pourquoi la menace de bombardement ne les a-t-elle pas dissuadés? Peut-on attribuer cet échec aux caractéristiques propres de la puissance aérienne? La dissuasion comporte plusieurs aspects, mais elle est profondément affectée par des considérations proprement militaires. Les dirigeants politiques et militaires de la RFY ont dû s'interroger, non pas tant sur le fait que les pays de l'OTAN allaient répondre à leurs initiatives (ces pays avaient déjà répondu par les sanctions), mais sur l'efficience probable de cette réponse. Si les logiques de la dissuasion nucléaire, et leurs limites, sont bien connues, la dissuasion peut également être fondée sur des armements conventionnels, dont la puissance aérienne ${ }^{31}$. Les dirigeants de la RFY concevaient peut-être des frappes aériennes limitées et ont sous-estimé la possibilité de la poursuite de l'action jusqu'à une situation d'enlisement qui peut déboucher sur un autre type d'interaction stratégique, et donc sur une nouvelle définition des objectifs des uns et des autres. Puissance aérienne et point de rupture économique et social L'une des conceptions à la fois fondatrice et fondamentale de la puissance aérienne, en tous cas aux Etats-Unis et au Royaume-Uni, concerne la capacité à détruire la capacité sociale, économique et politique d'un adversaire à faire la guerre ${ }^{32}$. Au cours des années 1920, le général italien Giulio Douhet (1869-1930) dans Il dominio dell'aria (1921) avait préconisé la destruction, non pas principalement d'objectifs militaires, mais de cibles industrielles et même directement des populations civiles afin d'anéantir la volonté populaire de résistance ${ }^{33}$. Dans une version actualisée de la conception de Douhet, le colonel John Warden, planificateur des frappes aériennes contre l'Irak durant l'opération « tempête du désert » en 1991, a soutenu que la campagne aérienne devait considérer l'ennemi "en tant que système " $^{34}$. A ses yeux, l'entité stratégique comporte cinq cercles concentriques: au centre la direction, puis les fonctions organiques essentielles, les infrastructures, la population et enfin les forces déployées. Pour paralyser le système adverse, les forces aériennes doivent frapper au cœur du système. Dès lors, viser les forces militaires apparait comme une inutile diversion. Il s'agit de frapper 
exclusivement la "tête du serpent», pour reprendre les expressions de certains officiers de l'armée de l'air américaine. Détruire un centre nerveux essentiel permet l'effondrement du système. Mais quel est ce centre nerveux? Le bombardement stratégique peut viser quatre types de cibles distinctes: la population civile, l'infrastructure industrielle, les réseaux de transport, de communications et d'électricité qui lient les différentes composantes des sociétés, ou le leadership politique ${ }^{35}$. Quoiqu'il en soit, cette conception suppose qu'il est possible d'évaluer les cibles pertinentes en combinant plusieurs types de ressources économiques. On peut ainsi atteindre, le plus souvent de manière progressive, le point de rupture de l'adversaire: les coûts politiques, économiques et sociaux sont tels qu'ils l'obligent à abandonner la lutte. Les promoteurs du bombardement stratégique reconnaissent que cet usage de la puissance aérienne peut susciter un renforcement de la détermination des dirigeants et de la cohésion sociale. Mais ce renforcement est généralement temporaire et ne peut contrebalancer durablement l'ampleur des destructions et des difficultés de la vie quotidienne. Il reste cependant difficile de situer précisément le " point de rupture » de l'adversaire et dans certains cas exceptionnels, comme celui du Vietnam, ce point de rupture peut être inexistant ${ }^{36}$. Au cours des opérations au Kosovo, les officiers de l'armée de l'air américaine (notamment le général Michael Short) ont exprimé leur préférence pour la destruction de cibles "stratégiques", comme les ministères yougoslaves et les centrales électriques ${ }^{37}$. Le général Clark insistait, au contraire, pour que l'armée de l'air détruise les chars et l'artillerie serbe au Kosovo. L'un des thèmes récurrents des analyses publiques des généraux de l'armée de l'air américaine est qu'ils parviendraient effectivement à leurs objectifs dans les délais qu'ils avaient prévus, s'ils disposaient réellement de toutes les capacités qu'ils demandent et qu'aucune contrainte politique n'était imposée à leur action. Lors de l'audition des officiers généraux devant la commission des forces armées du Sénat le 21 octobre 1999, ils ont, comme souvent, regretté les contraintes qui ont, à leurs yeux, empêché, ne serait-ce que dans un premier temps parfois, le bombardement des cibles " politiquement sensibles », comme la télévision serbe, le réseau d'approvisionnement en électricité, certaines cibles situées au Monténégro (particulièrement le terrain d'aviation de Podgorica) et surtout des cibles situées dans Belgrade et aux alentours de la capitale. Le général Michael Short (Joint Forces Air Component Commander) a expliqué, en usant d'une métaphore couramment employée par les officiers de l'armée de l'air: «il existait des cibles dans Belgrade que je croyais être d'une importance stratégique (...) que je croyais être près de la tête du serpent que les Français nous ont empêché de frapper ${ }^{38}$. En fait, dès le début des opérations - et à coup sûr après le dixième jour de la guerre et le bombardement du ministère de l'Intérieur le 3 avril - les décideurs militaires américains ont pensé qu'il était nécessaire de combiner l'attaque du potentiel militaire (qui vise des cibles militaires: bunkers, casernes, dépôts de munitions) et celle du potentiel économique et social (usines, ponts, stations de télévision et installations électriques). L'objectif était alors d'infliger des dommages à la société serbe et à son potentiel économique pour toucher indirectement le leadership politique. Dans le cas du Kosovo, l'impact des bombardements sur le potentiel économique et sur la cohésion sociale en RFY et ses conséquences sur le leadership politique et militaire reste incertain. Cette conception de la puissance aérienne présuppose que le bombardement va provoquer un changement soudain dans les conditions de vie, sur le plan économique comme sur celui des relations sociales. Plus cet effet des bombardements est net, plus la probabilité qu'il permette d'affecter le 
leadership est élevée. Or, au moment du déclenchement des frappes, la RFY est déjà un pays qui connaît une situation économique catastrophique. En 1993, l'inflation était déjà galopante. De plus, des sanctions économiques ont été imposées à la République de Yougoslavie et étaient entrées en vigueur presqu'un an auparavant. Le 9 mars 1998, les ministres des Affaires Etrangères du groupe de contact (Allemagne, Etats-Unis, France, Grande- Bretagne, Italie, Russie) adoptent des sanctions économiques et diplomatiques à l'encontre de la RFY. Le 31 mars 1998, l'ONU adoptait la résolution 1160 qui prévoyait un embargo sur les armes à destination de la RFY. Les ministres des Affaires Etrangères des pays de l'Union Européenne annonçaient le 9 mai 1998, un embargo sur les investissements étrangers en RFY et un mois plus tard, le 8 juin 1998, lors du sommet de Luxembourg, les dirigeants politiques européens approuvaient l'interdiction de nouveaux investissements en Serbie et décidaient du gel des avoirs du gouvernement de Belgrade sur le territoire de l'Union Européenne. Le gouvernement des Etats-Unis adoptait des mesures identiques. Enfin, lors du sommet de Cardiff, les 15 et 16 juin 1998, les pays membres de l'Union Européenne décidaient d'interdire les liaisons aériennes assurées par les compagnies yougoslaves en Europe. De mai 1998 jusqu'au début des frappes aériennes, en mars 1999, près de onze mois se sont écoulés au cours desquels les sanctions économiques ont produit des effets sur l'économie et la société en RFY. Une situation économique catastrophique et des sanctions plus sévères encore mises en place pendant un an n'ont pas forcé les dirigeants serbes à plier. L'objectif de destruction lente, mais inexorable, de l'économie et de la fabrique sociale de la RFY pour les forcer à négocier était celui des sanctions économiques, tout autant et même davantage que celui des bombardements. Les évaluations, souvent peu rigoureuses, des sanctions, confondent ici le problème de l'impact politique des sanctions et celui de leurs effets économiques et sociaux, ainsi que la question des écarts éventuels entre les uns et les autres. Que des sanctions économiques ne parviennent pas à faire céder un groupe de leaders politiques ne veut pas dire qu'elles n'exercent aucune influence sur une économie et une société. De plus, dans ce contexte, le bombardement du potentiel économique et social de la RFY est apparu, en tous cas jusqu'aux dernières semaines de la crise, discriminé, différent d'une destruction en règle des villes par exemple. Le bombardement des installations électriques, par exemple, a été volontairement conduit d'une manière telle que la réparation ne présentait pas de difficultés majeures. Il n'était pas question de détruire ces installations une fois pour toutes, mais plutôt d'exercer une pression graduelle sur l'adversaire. Au total, les destructions infligées par la stratégie aérienne de "punition » ont affecté l'économie et la société de la RFY, mais pas d'une manière telle qu'elles représentassent une rupture brutale et significative par rapport aux dommages pré-existants dus aux sanctions. La puissance aérienne face au potentiel militaire Dans une logique coercitive, la puissance aérienne peut viser les capacités militaires d'un adversaire ${ }^{39}$. Il s'agit d'attaquer ses forces militaires et de les affaiblir jusqu'au point où les forces terrestres peuvent occuper le terrain sans subir des dommages considérables. Seules comptent les capacités militaires. Il est inutile de détruire des bâtiments ou des ponts, il faut s'attaquer directement au potentiel militaire. Ce type de campagne aérienne prend pour cible les entreprises d'armement, les lignes d'approvisionnement entre le front et l'intérieur du pays concerné, les mouvements de troupes et les moyens de communication sur le théâtre d'opération, enfin directement les forces terrestres. Devant de telles destructions, l'adversaire n'est plus en mesure de parvenir à ses objectifs de conquête territoriale. On reste dans la logique coercitive, car ces actions ne sont pas nécessairement accompagnées de la 
conquête d'un territoire par exemple. La campagne aérienne que l'on peut appeler "d'interdiction " (contraire de "punition») se concentre sur la stratégie militaire de l'adversaire. Cette utilisation de la puissance aérienne n'aura pas le même effet selon les stratégies militaires adoptées par l'adversaire et toutes ne présentent pas les mêmes points faibles. Une stratégie fondée sur le combat mécanisé et une stratégie de guérilla n'ont évidemment pas les mêmes caractéristiques et l'interdiction aura tendance à être plus efficace contre la préparation d'opérations conventionnelles. L'utilisation de la puissance aérienne contre les forces militaires et policières de la RFY a été l'argument officiellement évoqué par Jacques Chirac dans son intervention du 29 mars 1999. Il soulignait qu'il fallait « enlever à ce régime les moyens dont il s'est doté pour conduire ces opérations (opérations d'épuration ethnique notamment, NDLR) (...) réduire les moyens de répression du président Milosevic $»^{40}$. En dehors de l'influence de la Russie, c'est également l'argument de l'affaiblissement matériel et moral du potentiel militaire et policier que Jacques Chirac a publiquement évoqué pour expliquer la capitulation des dirigeants politiques et militaires de la $\mathrm{RFY}^{41}$. L'évaluation de l'impact de la campagne aérienne sur le potentiel militaire de la RFY au Kosovo a naturellement constitué un enjeu important. D'autant que les unités de l'armée yougoslave, qui se sont retirées du Kosovo en onze jours, restaient plutôt bien équipées et, en apparence tout au moins, en état de combattre ${ }^{42}$. Entre la fin des hostilités en juin et la conférence de presse du général Wesley Clark du 16 septembre 1999, l'estimation officielle du nombre de chars détruits par les forces aériennes de l'OTAN a quelque peu diminué, tout en restant globalement dans les mêmes proportions, c'est-à-dire un tiers des chars déployés par la RFY au Kosovo. Au total, plusieurs évaluations ont été citées. Au cours des derniers jours du conflit en juin, les dirigeants de l'OTAN pensaient que les frappes aériennes avaient détruit 150 chars, les porte-parole du Pentagone ont annoncé peu après que le total était de 122, enfin le général Wesley Clark avait annoncé à la fin juin que les frappes aériennes en avait détruit $110^{43}$. Lors de la conférence de presse du 16 septembre 1999, le général Clark précisait qu'au total 93 chars serbes avaient été détruits, 26 épaves avaient été effectivement retrouvées et les renseignements collectés indiquaient que 67 autres blindés avaient été directement atteints et que ces épaves avaient été retirées du champ de bataille par les Serbes, une procédure classique dans toutes les armées du monde, surtout dans ce contexte ${ }^{44}$. Au cours de cette conférence de presse, le général Clark précisait que les forces aériennes avaient détruit en outre 153 transports de troupes blindés (l'estimation première était de 216), 339 véhicules militaires et 389 pièces d'artillerie et mortiers. L'évaluation de ces destructions dans l'absolu n'a, en réalité, guère de sens. L'utilisation de la destruction de chars comme indice principal de l'affaiblissement du potentiel militaire de la RFY n'est pertinent que si l'on raisonne dans le cadre d'un affrontement conventionnel classique dans lequel le char reste un système d'arme essentiel, notamment parce que sa mobilité et sa puissance de feu permettent la percée. Même si l'on accepte ce critère, la campagne aérienne contre le potentiel militaire et policier de la RFY au Kosovo a révélé, une fois de plus, combien la dispersion, le camouflage et les leurres pouvaient réduire considérablement les pertes face à une puissance de feu destructrice. Cela ne veut pas dire que la campagne aérienne ait été sans effet: elle a obligé les forces serbes à se disperser et à s'enterrer pour se protéger, ce qui les a paralysé. L'action des forces militaires et paramilitaires de la RFY au Kosovo s'apparentait pour partie à des opérations de contre-guérilla et pour partie à un effort pour vider le Kosovo des Kosovars. Il s'agissait d'expulser les populations et de détruire des villages afin que les 
combattants de l'armée de libération du Kosovo ne puissent y trouver le soutien dont ils avaient besoin. Ces opérations comprenaient effectivement l'action de véhicules blindés et de l'artillerie, mais il ne s'agissait pas d'un combat mécanisé classique. Les besoins en logistique et en communication étaient limités. De plus, comme les unités individuelles pouvaient agir de manière relativement autonome, seuls des réseaux de communication rudimentaires étaient nécessaires. Contre un tel adversaire, la stratégie d'interdiction par la puissance aérienne est plus difficile à mettre en œuvre et ses résultats sont plus imprévisibles. Certains généraux de l'armée de l'air américaine, notamment le général Leaf, ont également souligné que ce décompte des chars était sans objet, mais pour d'autres raisons. Il a expliqué que ces chars retournaient dans un pays affaibli, dont l'infrastructure militaire avait subi d'importantes destructions. Dépourvus de l'infrastructure adéquate, ces chars ne pourraient être d'une grande utilité 45 . Pour comprendre l'efficacité relative de la coercition par la destruction du potentiel militaire, l'évaluation pertinente demeure celle des dirigeants politiques et militaires serbes au cours du conflit. En d'autres termes, les chiffres de destruction du matériel peuvent paraître faibles par rapport aux attentes exprimées par les chefs militaires de l'OTAN, ou aux moyens des pays concernés, mais c'est à l'aune du calcul des décideurs serbes que ces pertes sur le champ de bataille produisent ou non des effets. Il semble que les pertes des forces de la RFY en blindés aient été concentrées dans les dernières semaines de la campagne aérienne (approximativement $40 \%$ des pertes totales au cours de la dernière semaine et $80 \%$ dans les deux dernières semaines et demi) ${ }^{46}$. Si cette concentration a pu exercer une influence, au total, ce ne sont pas les destructions infligées à l'outil militaire yougoslave proprement dit qui ont amené les dirigeants politiques serbes à faire machine arrière. Appui au sol de l'UCK et guerre terrestre La guerre terrestre ne peut faire l'objet ici de longs développements. Il s'agit d'une stratégie militaire dite conventionnelle combinant le feu et le mouvement et qui aurait visé la conquête du Kosovo. Cette action des troupes au sol aurait été appuyée par la puissance aérienne. Cette utilisation de la puissance aérienne semblait initialement difficile à envisager du fait de l'absence de troupes au sol en mesure de désigner des cibles et surtout de débusquer l'adversaire. On a cependant pu penser que l'UCK, qui avait connu de sérieux revers, était sur le point de devenir, avec le soutien secret des pays de l'OTAN, une véritable menace militaire pour les Serbes. Son action forçant les unités militaires et paramilitaires serbes à manœuvrer, elle les exposait à la puissance aérienne. L'appui au sol a été effectivement utilisé au moins une fois pour soutenir une offensive de l'armée de libération du Kosovo (UCK). A la fin avril 1999, des agents de la CIA et des éléments des forces spéciales de l'armée de terre américaine avaient commencé à organiser les combattants de l'UCK ${ }^{47}$. Le 26 mai, ceux-ci lançaient une offensive avec le soutien de l'artillerie albanaise afin d'assurer une voie d'approvisionnement. En trois jours, leur offensive était repoussée par les forces serbes et les combattants de l'UCK subissaient à leur tour l'assaut des soldats yougoslaves sur le mont Pastrik, au sud du Kosovo. Considérant cette situation inacceptable le commandement de l'OTAN décidait de soutenir les combattants de l'UCK au sol. Le 7 juin, deux bombardiers B-52 lançaient une opération de soutien des troupes au sol et nombre d'officiers de l'OTAN pensaient alors que les troupes serbes avaient subi des pertes considérables. Cette attaque, détruisant brutalement et en masse l'armée yougoslave aurait même pu être considérée comme un tournant conduisant les leaders politiques serbes à réaliser que leur instrument militaire (éventuellement utile pour se maintenir au pouvoir) pouvait connaître une rapide destruction. Or, sur le mont 
Pastrik, on n'a guère noté les signes de destructions majeures. Les évaluations des dommages de cette offensive après la fin de la campagne aérienne ont été plus modestes qu'initialement. Enfin, le dernier facteur est la menace d'une invasion terrestre par les forces de l'OTAN. En dépit de leurs déclarations réitérées, certains dirigeants politiques des pays membres de l'OTAN, notamment au Royaume-Uni, aux Etats-Unis, avaient entamé la planification d'une intervention terrestre de grande ampleur. Début juin, Tony Blair - le premier à réclamer publiquement une telle intervention - avait fait préparer un courrier destiné à mobiliser les réserves permettant l'engagement de 50000 soldats britanniques. Au cours de la deuxième moitié du mois de mai (et notamment le 18 mai), la rhétorique du président Clinton s'était également modifiée et il soulignait, pour la première fois, qu'il n'excluait aucune possibilité. La veille de la suspension des frappes, une équipe des principaux décideurs américains en matière de sécurité avait été réunie pour envisager la suite des événements. Au cours de cette réunion l'ordre des préférences des Etats-Unis - une victoire de l'OTAN, préserver la cohésion de l'alliance et maintenir une relation aussi bonne que possible avec la Russie - s'est transformé et la victoire est devenue le seul objectif décisif. Une réunion entre le président Clinton et les chefs d'état-major était prévue le 3 juin. D'après les notes de certains participants, Samuel «Sandy " Berger, conseiller du président Clinton pour la sécurité nationale, avait souligné « quatre faits irréductibles. Premièrement, nous allons gagner. Point. Jusqu'au bout. Il n'y a pas d'alternative. Deuxièmement, gagner signifie ce que nous disons que cela signifie. Troisièmement, la campagne aérienne a un sérieux impact. Quatrièmement, le président a dit qu'il n'excluait aucune option. Donc, revenez au premier point. Nous allons gagner $\aleph^{48}$. Si un consensus au sein de l'alliance s'était révélé impossible, la guerre terrestre aurait pu être menée par les Etats-Unis et le Royaume-Uni uniquement. Il est possible que Slobodan Milosevic ait finalement cru une telle intervention des forces terrestres possible. Les décideurs russes, en particulier Victor Tchernomirdin et Igor Ivanov, auraient été convaincus (peut-être par Strobe Talbott) de cette possibilité et auraient forcé Milosevic à ordonner la retraite de ses troupes. Une telle invasion aurait, en effet, placé la Russie dans une situation inextricable, contrainte de ne rien faire, ou d'aider les Serbes ce qui conduisait sans doute à l'escalade, mais aussi très probablement à la défaite des Serbes malgré tout. Cette hypothèse de l'influence russe sur Slobodan Milosevic peut contribuer à expliquer les frictions avec les décideurs russes qui pensaient peut-être extraire des concessions plus importantes de leur action. Enfin, il n'est pas à exclure qu'un accord secret ait été directement passé avec Milosevic, notamment pour l'assurer de la souveraineté et de l'intégrité territoriale de la RFY et de son maintien au pouvoir en dépit de l'action du tribunal pénal international. Dans ces conditions, la menace d'une invasion terrestre aurait joué un rôle dans la coercition que la puissance aérienne. Puissance aérienne et « rationalité stratégique limitée » dans la diplomatie coercitive Ces évaluations de la puissance aérienne permettent des distinctions utiles et parviennent à saisir certains résultats de son emploi au Kosovo. Cette crise semble plutôt révéler la faiblesse relative de la capacité coercitive des moyens aériens, qu'il s'agisse de la punition (Warden) ou de l'interdiction (Pape), ici renvoyées dos à dos. Ces conceptions de l'utilisation de l'arme aérienne, centrées sur l'impact militaire de l'arme aérienne, sont insuffisamment intégrées à la diplomatie coercitive. Elles ne tiennent pas suffisamment compte de sa complexité, de son ambiguïté, des incertitudes qu'elle comporte et des malentendus qu'elle peut entraîner. Elles négligent aussi certaines contributions 
importantes et spécifiques de la puissance aérienne qui ont joué un rôle dans la crise et son dénouement. S'il importe d'éclairer ces dynamiques au Kosovo, il faut aussi souligner les effets pervers potentiels de cet usage de la puissance aérienne. Puissance aérienne et diplomatie coercitive: quelle rationalité stratégique? Les conceptions stratégiques classiques utilisées pour analyser la contribution de la puissance aérienne dans ce contexte de diplomatie coercitive restent le plus souvent marquées par un modèle économique de la rationalité, la théorie de l'utilité espérée. Dans cette conception, chaque décideur politique rationnel possède une fonction d'utilité qui induit un ordre cohérent de préférences par rapport à l'ensemble bien défini d'options qui lui sont ouvertes. Il hiérarchise ces options selon le degré auquel elles favorisent ses objectifs et choisit toujours l'option dont l'utilité est la plus élevée. Si les limites de ce modèle ont été bien identifiées dans différents domaines, y compris en économie, et s'il existe des efforts élaborés pour mettre au jour et utiliser d'autres conceptions de la rationalité, l'analyse des réalités militaires (et de leurs rapports avec le politique) n'a pas suffisamment intégré ces apports développés en sociologie et en science politique. Ils sont pourtant indispensables pour comprendre les logiques de la puissance aérienne dans la diplomatie coercitive au Kosovo. La caractéristique essentielle de la conception classique de la rationalité économique est, en effet, de considérer les conditions du choix comme fixées et d'identifier la décision à la simple application d'un critère d'évaluation donné à un ensemble d'actions possibles également donné. Cette conception suppose que la procédure de formation de la volonté est sans importance : la réflexion et le calcul nécessaires à l'obtention de la solution n'apprennent rien de nouveau à l'individu, en particulier, il ne découvre rien sur ses propres préférences. Le sociologue et politiste Herbert Simon a avancé un autre modèle de rationalité : le modèle de la "rationalité limitée " ou "procédurale ${ }^{49}$. Les modèles de rationalité «procédurale » s'intéressent à la rationalité du processus du choix (et pas seulement aux résultats). Tout d'abord, cette conception procédurale met l'accent sur l'aspect délibératif de la décision : les conditions du choix, du côté des fins aussi bien que des moyens, ne sont pas données au décideur politique, mais font l'objet d'une recherche. De plus, les décideurs utilisent la méthode des buts intermédiaires. Il s'agit d'assigner des buts, pour lesquels on cherchera tour à tour les moyens les plus appropriés. Une fois ces moyens caractérisés, on les considérera à leur tour comme des buts dont il s'agira de déterminer les moyens de réalisation. Le moyen se transforme aisément en fin. Enfin, le principe de satisfaction joue un rôle éminent. Ce n'est pas l'action la meilleure relativement aux conditions objectives et subjectives de la décision, mais une action satisfaisante, dont l'évaluation est supérieure au niveau d'aspiration, ou seuil de satisfaction, de l'individu. Cette conception de la rationalité qui prend pour point de départ les capacités cognitives des individus peut être partiellement transposée au fonctionnement des organisations ${ }^{50}$. A partir de ce modèle, Charles Lindblom a proposé le modèle de l'incrémentalisme disjoint pour rendre compte d'un grand nombre d'actions publiques ${ }^{51}$. Les décideurs agissent lorsqu'ils en ont la possibilité, quand une occasion se présente. Ils traitent des problèmes relativement abordables, même s'ils ne sont que partiellement liés au problème central auquel il doivent faire face. Ils procèdent un pas à la fois et multiplient les coalitions avec d'autres joueurs sur de nombreux points. Leur gestion du temps est une ressource dans le jeu. Dans un système complexe, la transformation est progressive. Il s'agit d'écarter le pire et de minimiser les risques. Les logiques de la puissance aérienne au Kosovo: rationalité limitée et incrémentalisme disjoint Lorsque les décideurs politiques doivent prendre la décision 
d'utiliser la force armée, leurs informations sont fragmentaires et incomplètes. Or cette décision politique est souvent une décision urgente, ce qui interdit la recherche d'une information complète. L'ensemble des actions possibles n'est pas entièrement donné. Les options de recherche doivent être, elles-mêmes, recherchées. La puissance aérienne, employée de manière graduelle dans le cadre de la diplomatie coercitive, apparaît comme une composante importante du processus politico-militaire qui permet aux décideurs de découvrir progressivement des informations nouvelles sur leurs adversaires, sur leurs alliés, voire sur eux-mêmes, de préciser leurs préférences, à la fois leurs objectifs politiques et les missions des forces armées, et de les rendre progressivement plus cohérentes. La puissance aérienne est certes loin d'être l'arme absolue parfois décrite, mais elle est un instrument utile dans le cadre d'une rationalité stratégique limitée. C'est dans cette conception que l'on peut analyser de manière plus précise et réaliste ses atouts et ses limites. Même si cet impact est lui-même problématique, il peut néanmoins, à certaines conditions, jouer un rôle majeur dans les crises internationales aujourd'hui. La crise du Kosovo a marqué un retour réussi - un peu à la surprise générale - de la diplomatie coercitive fondée sur la puissance aérienne. Seuls les effets potentiellement pervers de cette utilisation de la force avaient été jusqu'à présent identifiés et dénoncés, au cours de la guerre du Vietnam par exemple, mais également dès le début des frappes aériennes au Kosovo. Les préférences et les fonctions d'utilité des décideurs politiques se précisent dans et par la confrontation avec les possibilités de choix, mais également avec les premières utilisations matérielles des "instruments " à leur disposition. La "volonté politique » ne se décrète pas. Elle se construit dans l'interaction stratégique, y compris si cette interaction est violente. Cette volonté est fabriquée et non pas spontanée. Les dirigeants politiques et militaires de l'OTAN pensaient initialement que deux ou trois jours de bombardements suffiraient à faire reculer Slobodan Milosevic, notamment parce que plusieurs d'entre eux avaient tiré de mauvais enseignements de l'opération «Deliberate force». Lorsque la réponse des troupes de la RFY a été d'accélérer l'offensive, les officiers de l'OTAN ont dû rapidement transformer leurs plans initiaux. La planification d'une campagne aérienne contre la RFY date de juin 1998 et comportait 40 scénarios de guerre aérienne dont certains récusaient la possibilité du «tout aérien ». La conception initiale de la campagne aérienne finalement retenue comportait trois phases: dans un premier temps, les forces aériennes frapperaient les défenses antiaériennes, les bunkers de commandement, les stations radar, les centres de communication de l'armée de la RFY. Il s'agissait d'obtenir la maîtrise de l'espace aérien. A l'issue de cette première phase, les dirigeants de l'OTAN, notamment Javier Solana, pensaient qu'une pause permettrait d'évaluer les effets militaires et politiques de ces frappes. Au cours d'une deuxième phase, l'arme aérienne serait dirigée contre les potentiels militaire et policier de la RFY : destruction des casernes, des éventuelles concentrations de chars, des voies de communication. Les forces aériennes viseraient également la logistique de l'armée yougoslave. Enfin, au cours d'une troisième phase seulement, les forces aériennes de l'alliance frapperaient des cibles dans la capitale de la RFY. Cette conception était celle qui avait été retenue et - sans doute pour signaler la détermination nouvelle du président Clinton et des dirigeants politiques des pays membres de l'OTAN - présentée publiquement à plusieurs journalistes le 11 septembre $1998^{52}$. En fait, dès les premiers jours de la campagne et, selon certaines informations, d'emblée, ce sont toutes les cibles qui seront visées à la fois. Ces usages ont été imbriqués. L'emploi de la puissance aérienne a rapidement signifié s'attaquer à tout en 
même temps, autant que les moyens disponibles le permettaient. Initialement, il existait une forte prohibition concernant les cibles civiles et la perte de pilotes et d'appareils. L'augmentation progressive du nombre de cibles a conduit à une progressive augmentation des dommages collatéraux. Même si le schéma initial n'a pas été respecté, la campagne aérienne a bien été graduelle. A la mi-avril, le général Clark demandait 300 avions supplémentaires afin de détruire un plus grand nombre de cibles militaires et à la fin du mois, les frappes se produisent vingt-quatre heures sur vingtquatre et visent systématiquement le potentiel stratégique de la RFY. Enfin, à partir de la mi-mai, plus de 170 appareils supplémentaires, s'ajoutant aux 650 déjà présents, sont déployés en Turquie et en Hongrie. Une progressive escalade s'est donc produite au cours de la crise. C'est la puissance aérienne utilisée de manière progressive, et pour régler très partiellement un problème précis - la volonté de Slobodan Milosevic - mais bien différent du problème central et plus urgent - l'action d'expulsion des Kosovars qui a progressivement rendu possible la planification de l'invasion du Kosovo par l'OTAN. La puissance aérienne n'agit pas seulement sur la psychologie de la cible. Elle peut conduire à un changement psychologique imprévu du côté de ceux qui l'utilisent. La volonté de parvenir à un résultat rapide était ce qui conduisait les conseillers militaires à déconseiller, en 1994-95, le recours à l'arme aérienne. Des frappes précises et efficaces sont difficiles à réaliser contre des miliciens qui utilisent des mortiers et se déplacent rapidement. Les chefs militaires savent - et ont souligné - que les frappes aériennes seules ne parviendraient pas à faire cesser l'action des troupes de la RFY. Leur conclusion était que, de ce fait, la puissance aérienne était inutilisable, voire créatrice d'effets pervers en accélérant le processus. Ils omettaient d'intégrer une logique politique, faisant de l'arme aérienne une étape indispensable pour passer à d'autres options. Certaines actions militaires peuvent se révéler nécessaires pour rendre concevable et justifier politiquement d'autres actions militaires. On peut alors parler de l'effet politique de la puissance aérienne en un sens global. Les arguments de John Warden et Robert Pape insistent sur des moyens spécifiques de parvenir au succès avec la puissance aérienne. Mais c'est aussi dans une perspective politico-diplomatique plus large que l'on peut aussi évaluer l'apport éventuel de la puissance aérienne. L'arme aérienne permet à la fois de parvenir initialement à un plus petit commun dénominateur entre 19 alliés et après son emploi graduel, elle peut en amener certains au moins à un consensus sur la nécessité d'employer d'autres moyens militaires. Dans un premier temps, les décideurs utilisent l'arme aérienne comme un instrument permettant d'agir, tout en préservant un consensus. Mais au fil du temps, cet « instrument » impose ses propres logiques. L'objection habituelle à ce type d'action est le danger d'enlisement. Cet enlisement est pourtant politiquement utile dans certaines circonstances. En un sens étroit et littéral, les arguments de Warden et de Pape semblent remis en cause par la crise du Kosovo, mais il n'en demeure pas moins que la puissance aérienne entendue comme une arme politique peut conduire les acteurs à atteindre un nouveau point dans leurs préférences stratégiques. Sans l'emploi graduel de la puissance aérienne, ils ne parviennent pas à ce nouveau point d'équilibre. La conception de Warden, celle des cercles concentriques répartis dans l'espace, doit être complétée par des cercles concentriques dans le temps, qui tiennent compte du caractère séquentiel des décisions et de la rationalité limitée des décideurs politiques et militaires. Pour le dire encore autrement, la puissance aérienne mise en œuvre graduellement permet en quelque sorte de fabriquer, de créer, une "dépendance du chemin ». La puissance aérienne apparaît bien comme l'arme de "l'incrémentalisme 
disjoint » et de la rationalité stratégique limitée ${ }^{53}$. Sa mise en œuvre est rapide, elle est flexible : on peut aisément arrêter, puis reprendre. Elle permet des ajustements. Les technologies permettent de minimiser les pertes pour soi et pour les populations civiles de l'adversaire. Elle permet d'agir, de montrer que l'on agit, et en même temps de donner le temps nécessaire pour réfléchir à d'autres options. Les décideurs politiques peuvent aussi contrôler plus aisément la puissance aérienne, ou croire plus aisément qu'ils le peuvent. Enfin, il s'agit d'un moyen d'action limité, il ne marque pas un engagement total. Par conséquent, l'impact proprement militaire (par la punition ou l'interdiction) peut être relativement modeste (ou indéterminé), alors même que l'instrument reste politiquement utile. Dans certains cas, la puissance aérienne est l'instrument de la transformation progressive des préférences politiques et de la fondation des coalitions nécessaires. Ce qui a été présenté comme un inconvénient potentiel de la puissance aérienne, pourrait bien être également, dans certaines circonstances, un atout ${ }^{54}$. La crise du Kosovo nous conduit à reprendre la notion de «bord du gouffre " (brinkmanship) développée par Thomas Schelling ${ }^{55}$. Le "bord du gouffre » est une situation caractérisée par la création d'un risque d'affrontement que le protagoniste qui prend l'initiative ne contrôle pas entièrement: "Le procédé tactique consiste (...) à laisser volontairement la situation se dégrader au point de ne plus pouvoir la maîtriser entièrement, dans l'espoir que la pression ainsi exercée sur l'adversaire sera suffisamment forte pour l'amener à résipiscence $»^{56}$. Dans le cas du Kosovo, les pays membres de l'OTAN ont créé, par la mise en œuvre de la puissance aérienne, une situation dans laquelle il existait un risque modéré mais progressivement croissant de guerre conventionnelle majeure. C'est l'incertitude créée par la puissance aérienne qui compte, plus que la certitude éventuelle qui l'accompagne sur le plan exclusivement militaire. Il existe une possibilité pour que la situation devienne incontrôlable. Si la puissance aérienne conduit ipso facto à un conflit majeur, il ne s'agit pas d'une opération de diplomatie coercitive: le conflit armé débuterait d'emblée. Inversement, s'il est certain que la puissance aérienne ne conduira en aucun cas à une guerre conventionnelle majeure, elle ne constituera que du «bruit » et non un signal. Elle n'imposerait aucun risque et ne démontrerait aucune volonté d'encourir certains risques. La puissance aérienne comporte, outre ses destructions propres, un risque plus important encore de voir une guerre majeure se déclencher. La manière dont cette guerre se produirait n'est pas prévisible. Les dirigeants politiques et militaires de l'OTAN ont créé un risque coercitif de plus en plus marqué qui conduisait à leur engagement toujours plus grand dont les conséquences étaient imprévisibles. Les dangers de la situation de «bord du gouffre » ont été souvent soulignés. Non seulement la manipulation du risque peut se révéler dangereuse pour celui qui veut manipuler, mais de plus l'engagement graduel peut conduire à un enlisement progressif dans une situation inextricable, un peu à l'image des Etats-Unis au Vietnam. La mise en œuvre de la puissance aérienne au cours de la crise du Kosovo a montré qu'un enlisement aérien «au bord du gouffre» pouvait produire des effets bénéfiques non prévus. Ces contributions, si réelles et sous-estimées soient-elles, ne font pas de l'arme aérienne une arme sans défauts. Il faut revenir à grands traits sur les effets pervers potentiels de la rationalité stratégique limitée de la puissance aérienne. D'abord, la flexibilité peut signifier une utilisation excessive et mal maîtrisée. Le fait de disposer de l'instrument incite à son utilisation systématique. Une telle utilisation comporte des risques d'escalade et de contagion. L'arme aérienne peut, comme au Vietnam, entraîner de graves confusions sur le centre de gravité et la psychologie des sociétés démocratiques, 
comme des adversaires potentiels. On ne badine donc pas impunément avec la force ${ }^{57}$. Vertus de l'enlisement " au bord du gouffre »? Ce n'est pas la première fois qu'une campagne aérienne prend un tour inattendu et que des processus qui semblent conduire à l'échec ou au demi-succès finissent par entraîner des conséquences imprévues et non voulues qui amènent à certains succès ${ }^{58}$. Depuis la fin de la Guerre Froide, un nouveau débat sur le déclin de l'utilité et des usages de la force armée dans les relations internationales s'est engagé. Ces débats sont remarquablement cycliques, même s'ils n'obéissent pas tous aux mêmes logiques. Du milieu des années 1950 aux années 1970, la grande querelle sur l'utilité de la force dans les relations internationales était liée au fait nucléaire. Il s'agissait de savoir si les armes nucléaires ne mettaient pas un terme à l'utilisation de la force comme instrument politique, tant les risques d'escalades étaient grands et ses conséquences éventuelles dramatiques ${ }^{59}$. L'interdépendance des années 1970 a également suscité des discussions sur la moindre utilité des armes notamment du fait de la multiplication des échanges commerciaux. Que l'art de la guerre devienne toujours plus sophistiqué sur le plan technologique et toujours moins héroïque - encore qu'il faille s'entendre ici sur une définition - ne signifie pas que le recours à la force soit derrière nous, au contraire. On mentionnera pour finir quelques enseignements complémentaires. La crise du Kosovo devrait permettre de mieux circonscrire les débats, dans lesquels le spectaculaire le dispute souvent au superficiel, sur la révolution dans les affaires militaires. Elle nous incite à mieux évaluer l'apport des transformations technologiques, même s'il est parfois exagéré, tout en reconnaissant que la plupart des aspects fondamentaux de cette crise n'avaient rien de révolutionnaire. Le scepticisme concernant les annonces régulières de transformations majeures (comme la révolution dans les affaires militaires) ne saurait être interprété, du point de vue du fonctionnement des organisations militaires, comme la reprise d'une position conservatrice. Le recours à la puissance aérienne au Kosovo révèle au contraire l'importance de la préparation des forces, de l'entraînement, de la coordination interarmées, de la formation des personnels, de la planification et de la réflexion en temps de paix, et ce quand bien même les évolutions seraient différentes au cours des opérations. Tous ses aspects comportent des réformes, des adaptations, un durcissement des exigences professionnelles. L'un des défis essentiels de l'art de la guerre aujourd'hui demeure la coordination rapide et efficace des armes et des armées au niveau opératif et tactique et la coordination des logiques politiques et militaires au niveau stratégique. Ces coordinations sont d'une complexité telle que peu d'armées au monde sont en mesure de les réaliser. Elles reposent sur des capacités humaines et organisationnelles de premier plan. La présente analyse montre que les sciences sociales, en particulier la sociologie et la science politique, peuvent éclairer ces interactions, ainsi que leurs effets sur l'efficience des armées. Elles contribuent ainsi à une meilleure intelligence de l'art de la guerre sous tous ses aspects. La diplomatie coercitive ne semble pas prête de disparaître des réalités internationales, pas plus que les usages politiques de la force armée. Bibliographie sélective

2 Sur la "diplomatie de la violence », Schelling T. C., Arms and Influence, New Haven, Yale University Press, 1966, 293 p. On complétera ces réflexions par, George A. L., Simons W. E., dir., The Limits of Coercive Diplomacy (1ère éd. 1971), Boulder, CO, Westview Press, 1994, 310 p. Pour une critique des arguments de Schelling, Hassner P., "On ne badine pas avec la force", Revue française de science politique, 21 (6), décembre 1971, pp. 1207- 1234. Sur l'évolution historique du bombardement, Facon P., Le bombardement stratégique, Monaco, Editions du Rocher- L'art de la guerre, 1995. 
Voir également, Warden J., La campagne aérienne. Planification en vue du combat (1ère éd. 1988), Paris, Economica- Bibliothèque stratégique, 1998, 206 p. Sur les constructions sociales et politiques de la puissance aérienne, Vennesson P., Les chevaliers de l'air. Aviation et conflit au XXème siècle, Paris, Presses de Science Po, 1997, 210 p. Sur la coercition aérienne, Pape R. A., Bombing to Win. Air Power and Coercion in War, Ithaca, Cornell University Press, 1996. Et les débats qu'il a suscité, Robert A. Pape, «The Limits of Precision- Guided Air Power », Security Studies, 7 (2), hiver 1997- 98, pp. 93- 114 ; Barry D. Watts, "Ignoring Reality. Problems of Theory and Evidence in Security Studies ", Security Studies, 7 (2), Hiver 1997- 98, pp. 115- 171; John A. Warden III, "Success in Modern War. A Response to Robert Pape's Bombing to Win ", Security Studies, 7 (2), Hiver 1997- 98, pp. 172- 190 ; Robert A. Pape, «The Air Force Strikes Back : A Reply to Barry Watts and John Warden ", Security Studies, 7 (2), hiver 1997- 98, pp. 191- 214; Karl Mueller, "Strategies of Coercion: Denial, Punishment, and the Future of Air Power », Security Studies, 7 (3), printemps 1998, pp. 182-228.

\section{NOTES}

1. Je remercie ceux qui ont complété ma documentation et qui ont bien voulu m'écouter tester sur eux certaines des idées présentées ici. Les commentaires et suggestions de Jean-Yves Haine et Arnaud Mercier m'ont été d'une grande utilité, même quand nous n'étions pas d'accord. Une version antérieure a été présentée à la conférence " Après le Kosovo : puissance aérospatiale et contrôle stratégique », Centre d'analyse sur la sécurité européenne, Paris, 18 janvier 2000. Marie Le Gonidec de Kerhalic a été une excellente collaboratrice de recherche. Ce texte n'engage que son auteur.

2. Cf. par exemple, Adam, B., dir., La guerre du Kosovo. Eclairages et commentaires, Bruxelles, GRIP-Complexe, 1999 ; « Fallout from Kosovo », Foreign Affairs, 78 (4), juilletaoût 1999 ; Hassner, P., « Guerre sans morts ou morts sans guerre? Les paradoxes de l'intervention au Kosovo ", Critique internationale, 4, été 1999, pp. 6-13.

3. Nous laissons de côté ce qui concerne la "guerre de l'information » et sa place dans la coercition. Sur ce thème en général, Feaver, P. D., «Blowback : Information Warfare and the Dynamics of Coercion ", Security Studies, 7 (4), été 1998, pp. 88-120. Sur les limites de la guerre de l'information au Kosovo, Timothy L. Thomas, « Kosovo and the Current Myth of Information Superiority », Parameters, 30 (1), printemps 2000, pp. 13-29.

4. Par exemple, en France, Les enseignements du Kosovo, Ministère de la défense, Analyses et références, novembre 1999 ; en Chine, Bodansky, Y., " Beijing and the Kosovo Crisis », Defense and Foreign Affairs Strategic Policy, 5-6, 1999, pp. 4-12. 5. Il n'est pas possible de développer ici une longue justification de ce découpage. Il suffit de noter qu'il ne s'agit pas là d'un choix effectué faute de mieux : l'argument qui sous-tend ce choix est bien que cette dimension proprement militaire et son interaction avec le politique est déterminante. 
6. Il faudrait reconstituer les rôles divers et substantiels de la puissance aérienne dans la « culture » de la sécurité nationale aux Etats-Unis où la puissance aérienne est une mystique, au point qu'on pourrait dire comme Daniel Ellsberg au moment de la guerre du Vietnam, que «bombarder est aussi américain que le gâteau aux cerises » ( " bombing is as American as cherry pie »). Ellsberg, D., "The American Way of War » (1ère éd. 1968), p. 253, dans Ellsberg, D., Papers on the War, New York, Pocket Books, 1972. Cf. Builder, C. E., The Icarus Syndrome. The Role of Air Power Theory in the Evolution and Fate of the U. S. Air Force, New Brunswick, Transaction Publishers, 1994 ; Noël, J.-C., Vers d'autres cieux. L'United States Air Force et le retournement du monde, Mémoire IEP, Paris, 1997.

7. IISS, The Military Balance, 1999-2000, Londres, IISS, 1999, p. 289.

8. Cohen, E. A., « The Meaning and Future of Air Power », Orbis, printemps 1995, p. 190. Nous reprendrons ici, sans plus de discussion dans les limites de cette analyse, le vocabulaire technique utilisé par les acteurs (frappes aériennes, coercition, appui au sol, etc.). Ce vocabulaire contribue certainement à construire la réalité (sans aucun doute ici à l'euphémiser) et, en ce sens, il n'est pas neutre. Cette caractéristique a été repérée de longue date notamment pour ce qui concerne le vocabulaire de la dissuasion nucléaire (cf. par exemple, Cohn, C., « Sex and Death in the Rational World of Defense Intellectuals », Signs : Journal of Women in Culture and Society, 12 (4), 1987, pp. 687-718). Toutefois, en ce domaine, les effets du langage paraissent assez indéterminés. De plus, l'utilisation d'un langage technique euphémisé est courant dans les professions en contact direct avec les destructions, les blessures et la mort, comme la police, les pompiers, les médecins. Un tel vocabulaire apparaitt comme la condition nécessaire à l'exercice de certains métiers. On peut se demander si la sociologie politique de ces professions peut aisément s'en affranchir. Sur le rôle du vocabulaire bureaucratique dans la banalisation du mal, Arendt, H., Eichman à Jérusalem. Rapport sur la banalité du mal (1ère éd. 1963), Paris, Gallimard-folio-histoire, 1991, p. 118, 144-145.

9. «L'appui au sol consiste à utiliser l'arme aérienne dans la bataille terrestre, pour empêcher l'ennemi d'utiliser librement ses forces combattantes, aussi bien dans la zone des combats qu'à l'arrière ». En France, on évoque « l'action aérienne dans la zone de contact et dans la profondeur ». Le bombardement stratégique désigne « l'attaque du territoire ennemi au-delà du théâtre des opérations ", qu'il s'agisse d'objectifs économiques, sociaux ou militaires. Coutau-Bégarie, H., Traité de stratégie, Paris, Economica-Perspectives stratégiques, 1999, pp. 607-612, 616-618. Sur les origines de l'appui au sol, Hallion, R. P., Strike from the Sky. The History of Battlefield Air Attack, 1911-1945, Shrewsbury, Airlife Publishing-Smithsonian, History of Aviation Series, 1989.

10. Rousset, V. \& Wodka-Gallien, P., « La couverture du flanc sud », Air Zone Magazine, 27, septembre-octobre 1999, pp. 24-31.

11. Gordon, M. R. \& Trainor, Bernard E., The General's War. The Inside Story of the Conflict in the Gulf, Boston, Little, Brown and Company, 1995 ; Woodward, B., Chefs de guerre, Paris, Calmann-Lévy, 1991, pp. 331-333.

12. Biddle, S., «Victory Misunderstood : What the Gulf War Tells Us about the Future of Conflict », International Security, 21 (2), automne 1996, pp. 139-179.

13. Rostow, W. W., Pre-Invasion Bombing Strategy. General Eisenhower Decision of March 25, 1944, Austin, University of Texas Press, 1981, pp. 3-6. L'économiste Walt Rostow, plus connu en science économique pour ses recherches sur les étapes de la croissance, a participé au processus de décision qu'il évoque dans cet ouvrage (tout 
comme dans les autres monographies de la même série qu'il a consacré à plusieurs décisions politiques), ce qui bien sûr affecte son analyse. Rostow était (et est resté) favorable à la destruction des champs pétrolifères et critique l'option centrée sur le transport ferroviaire.

14. Galbraith, J. K., "Patient Firmness Is a Better Policy than Bombing », International Herald Tribune, 26 avril 1999, p. 8.

15. Mierzejewski, A. C., The Collapse of the German War Economy, 1944-1945. Allied Air Power and the German National Railway, Chapel Hill, The University of North Carolina Press, 1988, pp. 162-176, 177-187 ; Murray, W., « Reflections on the Combined Bomber Offensive », Militärgeschichtliche Mitteilungen, 51, 1992, pp. 73-94 ; Biddle, T. D., «British and American Approaches to Strategic Bombing: Their Origins and Implementation in the World War II Combined Bomber Offensive ", dans Gooch, J., Airpower. Theory and Practice, Londres, Frank Cass, 1995, pp. 91-144 ; Overy, R. J., The Air War, 1939-1945, New York, Stein and Day, 1980 ; Overy, R. J., Why the Allies Won, New York, W.W. Norton \& Company, 1995, pp. 101-133. Sur l'évolution historique du bombardement, Facon P., Le bombardement stratégique, Monaco, Editions du RocherL'art de la guerre, 1995. Pour une réflexion globale sur l'évaluation politique et sociale des bombardements, Quester, G. H., « The Psychological Effects of Bombing on Civilian Populations : Wars of the Past », Glad, B., dir., Psychological Dimensions of War, Newbury Park, Sage, 1990, pp. 200-214.

16. Werrell, K. P., Blankets of Fire. U.S. Bombers over Japan during World War II, Washington, Smithsonian Institution Press, 1996, pp. 224-241. Pour une interprétation critique du bombardement stratégique du Japon, Pape, R. A., Bombing to Win. Air Power and Coercion in War, Ithaca, Cornell University Press, 1996, pp. 87-136.

17. Les auteurs qui ont défini et mis en oeuvre la notion de diplomatie coercitive (sans développer un point de vue tout à fait identique à son propos) sont : Schelling, T. C., Arms and Influence, New Haven, CT, Yale University Press, 1996 ; George, A. L. \& Simons, W. E., dir., The Limits of Coercive Diplomacy (1ère éd. 1971), Boulder, Westview Press, 1994 (la nouvelle édition a été amputée du premier chapitre, pourtant utile, de l'ancienne). Pour une application par George lui-même à la guerre du Golfe, George, A. L., Forceful Persuasion : Coercive Diplomacy as an Alternative to War, Washington, D.C., United States Institute for Peace, 1991. Pour une critique, Hassner, P., « On ne badine pas avec la force ", Revue française de science politique, 21 (6), 1971, pp. 1207-1234. Pour un effort d'actualisation, qui reste en deçà des précurseurs, Freedman, L., dir., Strategic Coercion. Concepts and Cases, Oxford, Oxford University Press, 1998.

18. Poirier, L., Des stratégies nucléaires (1ère éd. 1977), Bruxelles, Complexe, 1988, p. 132 ; Poirier, L., « Dissuasion (stratégie de) », p. 182, dans Chaliand, G. \& Blin, A., dir., Dictionnaire de stratégie militaire, Paris, Perrin, 1998 ; Shelling, T. C., Stratégie du conflit (1ère éd. 1960), Paris, PUF-Perspectives internationales, 1986, pp. 240-244 ; Schelling, T. C., Arms and Influence, op. cit., pp. 69-78, 170-176. Il est possible de définir la notion de « compellence » en excluant tout usage de la force. Ce qui est ainsi gagné en clarté appauvrit trop la notion. C'est pourquoi nous adoptons ici la conception de Schelling. Pour un effort de clarification qui écarte l'utilisation de la force (et que nous ne retiendrons donc pas), Schaub, G. Jr., "Compellence : Resuscitating the Concept », pp. 40-46, dans Freedman, L., dir., op. cit, 1998.

19. Coutau-Bégarie, H., Traité de stratégie, Paris, Economica-Perspectives stratégiques, 1999, p. 408.

20. Schelling, T. C., Arms and Influence, op. cit., p. 3. 
21. Ellsberg, D., «The Theory and Practice of Blackmail » (1ère éd. 1968), dans Young, O. R., dir., Bargaining : Formal Theories of Negotiation, Urbana, University of Illinois Press, 1975. Voir également, Osgood, R. E. \& Tucker, R. W., Force, Order, and Justice, Baltimore, The Johns Hopkins Press, 1967, pp. 3-192 ; Jervis, R., « Bargaining and Bargaining Tactics ", dans Pennock, J. R. \& Chapman, J. W., dir., Coercion. Nomos XIV. Yearbook of the American Society for Political and Legal Philosophy, Chicago, Aldine, 1972, pp. 272-288 ; Snyder, G. H. \& Diesing, P., Conflict Among Nations. Bargaining Decision Making, and System Structure in International Crises, Princeton, N.J., Princeton University Press, 1977, pp. 183-281.

22. George, A. L., « The Development of Doctrine and Strategy », dans George, A. L., Hall, D. K., Simons, W. E., The Limits of Coercive Diplomacy. Laos, Cuba, Vietnam, Boston, Little, Brown and Company, 1971, pp. 16-35.

23. Schelling, Arms and Influence, op. cit., p. 2.

24. Lauren, P. G., "Coercive Diplomacy and Ultimata : Theory and Practice in History ", dans George, A. L. \& Simons, W. E., The Limits of Coercive Diplomacy, Boulder, Westview Press, 1994, pp. 23-50 ; Mandel, R., « The Effectiveness of Gunboat Diplomacy », International Studies Quarterly, 30, 1986, pp. 59-76.

25. Sur les limites de la puissance aérienne au Vietnam, Clodfelter, M., The Limits of Air Power. The American Bombing of North Vietnam, New York, The Free Press, 1989 ; Tilford, E. H. Jr., Setup. What the Air Force Did in Vietnam and Why, Maxwell Air Force Base, AL, Air University Press, 1991. Sur la mise en oeuvre de la puissance aérienne après la guerre du Golfe : Shultz, R. H. Jr., "Compellence and the Role of Air Power as a Political Instrument ", dans Shultz, R. H. Jr. \& Pfaltzgraff, R. L. Jr., dir., The Future of Air Power in the Aftermath of the Gulf War, Maxwell Air Force Base, AL, Air University Press, 1992, pp. 171-191 ; Gates, D., « Air Power and the Theory and Practice of Coercion ", Defense Analysis, 13 (3), 1997, pp. 239-254.

26. Byman, D., Pollack, K., Waxman, M., « Coercing Saddam Hussein : Lessons from the Past », Survival, 40 (3), automne 1998, pp. 127-151. Pour une critique de la politique des Etats-Unis vis-à-vis de l'Irak, Salamé, G., « Irak : une déraison politiquement correcte », Critique internationale, 2, hiver 1999, pp. 6-14.

27. Cité par Ignatieff, M., " The Virtual Commander », The New Yorker, 2 août 1999, p. 32.

28. Il existe un riche ensemble de travaux sur la dimension normative de la guerre aérienne. L'ouvrage classique de Michael Walzer fournit une bonne entrée en matière, mais sa lecture de l'histoire du bombardement stratégique reste superficielle : Walzer, M., Guerres justes et injustes (1ère éd. 1977), Paris, Belin, 1999. Pour plus de précisions, Schaffer, R., Wings of Judgment, New York, Oxford, 1985 ; Sherry, M., The Rise of American Air Power, New Haven, Conn., Yale University Press, 1987 ; Crane, C., Bombs, Cities and Civilians, Lawrence, Kansas University Press, 1993. Sur les rapports entre la puissance aérienne et le droit des conflits armés : Biddle, T. D., «Air Power », dans Howard, M., Andreopoulos, G. J., Shulman, M. R., dir., The Laws of War. Constraints on Warfare in the Western World, New Haven, Yale University Press, 1994, pp. 140-159. 29. Châtelot, C., « La communauté internationale lance un nouvel avertissement à Slobodan Milosevic », Le Monde, 24 septembre 1998, p. 5 ; Myers, S. L., « NATO Raises Pressure on Serbs, Dangling Specter of Kosovo Intervention ", International Herald Tribune, 23 septembre 1998, p. 6 ; Whitney, C. R., «NATO Takes First Step Toward Military Action in Kosovo », International Herald Tribune, 25 septembre 1998, p. 6. 
30. Holbrooke, R., To End a War (1ère éd. 1998), New York, The Modern Library, 1999, pp. 106-107, 142-152 ; Ripley, T., Operation Deliberate Force, Londres, Centre for Defence and International Security Studies, 1999.

31. Mearsheimer, J., Conventional Deterrence, Ithaca, NY, Cornell University Press, 1983, pp. 23-66. Sur les logiques dissuasives et coercitives au cours de la crise puis de la guerre du Golfe, Stein, J. G., « Deterrence and Compellence in the Gulf, 1990-91 : A Failed or Impossible Task? », International Security, 17 (2), automne 1992, 147-179 ; Herrmann, R., « Coercive Diplomacy and the Crisis over Kuwait », 229-264, dans George, A. L., Simons, W. E., dir., The Limits of Coercive Diplomacy, Boulder, Westview Press, 1994.

32. Pour d'autres modèles, Vennesson P., Les chevaliers de l'air. Aviation et conflit au XXème siècle, Paris, Presses de Science Po, 1997.

33. Warner, E., « Douhet, Mitchell, Severski : les théories de la guerre aérienne », dans Mead Earl, E., dir., Les maîtres de la stratégie vol. 2 (1ère éd. 1943), Paris, Flammarion Champs, 1987, pp. 250-260.

34. Warden III, J., « L'ennemi en tant que système » (1ère éd. 1995), pp. 175-199, reproduit en appendice à Warden III, J., La campagne aérienne. Planification en vue du combat (1ère éd. 1988), Paris, Economica-Bibliothèque stratégique, 1998.

35. Depuis les premières attaques aériennes au cours de la Première Guerre Mondiale, jusqu'au Kosovo, le système électrique a constitué une cible classique des attaques aériennes, Kuehl, D. T., « Airpower vs. Electricity : Electric Power as a Target For Strategic Air Operations ", dans Gooch, J., Airpower. Theory and Practice, Londres, Frank Cass, 1995, pp. 237-266.

36. Pour un bon exposé de cette thèse, Mueller, J. E., " The Search of the " Breaking Point » in Vietnam. The Statistics of a Deadly Quarrel », dans Russett, B., Starr, H., Stoll, R. J., dir., Choices in World Politics. Sovereignty and Interdependence, New York, W. H. Freeman and Company, 1989, pp. 76-93.

37. Priest, D., « Strikes Divided NATO Chiefs », International Herald Tribune, 22 septembre 1999, p. 1.

38. Arkin, W. M., « Belgrade Hit Earlier than Previously Reported », Defense Daily, 27 octobre 1999, p. 4.

39. Pape, R. A., Bombing to Win. Air Power and Coercion in War, op. cit. ; Pape, R. A., "Coercion and Military Strategy : Why Denial Works and Punishment Doesn't ", The Journal of Strategic Studies, 15 (4), décembre 1992, pp. 423-475. C'est l'utilisation de la puissance aérienne que Robert Pape appelle « denial » (interdiction). Sa démonstration, par ailleurs solide et argumentée, repose sur une définition excessivement large de la notion « d'interdiction » et trop réductrice de la notion de « punition ». Dans ces conditions, il n'est pas difficile, mais pas non plus très incisif, de montrer que c'est l'interdiction et non la punition qui porte ses fruits. De plus, Pape étudie à la fois la coercition au cours de conflits armés et en dehors de ceux-ci, ce qui affecte ses résultats. Pour un débat sur ses thèses : Pape, R. A., « The Limits of Precision-Guided Air Power ", Security Studies, 7 (2), hiver 1997-98, pp. 93-114 ; Watts, B. D., « Ignoring Reality. Problems of Theory and Evidence in Security Studies », Security Studies, 7 (2), hiver 1997-98, pp. 115-171 ; Warden III, J. A., « Success in Modern War. A Response to Robert Pape's Bombing to Win », Security Studies, 7 (2), hiver 1997-98, pp. 172-190 ; Pape, R. A., "The Air Force Strikes Back : A Reply to Barry Watts and John Warden ", Security Studies, 7 (2), hiver 1997-98, pp. 191-214 ; Mueller, K., « Strategies of Coercion : 
Denial, Punishment, and the Future of Air Power », Security Studies, 7 (3), printemps 1998, pp. 182-228.

40. "Cela suffit », Libération, 30 mars 1999, p. 7.

41. Entretien du président Jacques Chirac à la chaîne de télévision française TF1, Paris, 10 juin 1999, dans Ministère des Affaires Etrangères, Documents d'actualité internationale, 14, 15 juillet 1999, Paris, La documentation française, 1999, p. 572.

42. On a pu compter quittant le Kosovo, outre 47000 soldats, policiers et membres de forces paramilitaires de la RFY, 220 chars, 300 véhicules blindés de transport de troupes, plus de 300 pièces d'artillerie, ansi que des centaines d'autres véhicules et des équipements militaires chargés sur camions. Myers, S. L., « NATO Air War May Have Done Less Damage Than Alliance Thought », International Herald Tribune, 29 juin 1999, p. 4.

43. Myers, S. L., " NATO Air War May Have Done Less Damage Than Alliance Thought », International Herald Tribune, 29 juin 1999, p. 4 ; Fitchett, J., « NATO Lowers Its Tally Of Tanks Hit in Kosovo ", International Herald Tribune, 17 septembre 1999, p. 5. En dehors de motifs de propagande, le décalage entre les estimations au cours du conflit et après la fin des hostilités tient principalement au fait que les pilotes croyaient parfois à tort avoir atteint leur cible et que, dans d'autres cas, des leurres avaient été pris pour cible. Des désaccords et des estimations très divergentes persistent cependant, Timothy L. Thomas, « Kosovo and the Current Myth of Information Superiority ", Parameters, 30 (1), printemps 2000 , pp. 18-23.

44. Fitchett, J., « NATO Lowers Its Tally Of Tanks Hit in Kosovo », International Herald Tribune, 17 septembre 1999, p. 5 ; Smith, M., « Nato rejects criticism of air war », Financial Times, 17 septembre 1999, p. 3. Une armée qui contrôle le terrain au sol est habituellement en mesure d'assurer la réparation de ses véhicules à l'arrière du front et/ou de récupérer les véhicules partiellement détruits pour utiliser les pièces détachées.

45. Cité par Ignatieff, M., « The Virtual Commander », The New Yorker, 2 août 1999, p. 35.

46. Estimation du Pentagone citée par, Daalder, I. H. \& O’Hanlon, M. E., « Unlearning the Lessons of Kosovo », Foreign Policy, automne 1999, p. 131.

47. Priest, D., « NATO Secretly Planned An Invasion of Kosovo », International Herald Tribune, 20 septembre 1999, p. 5.

48. Erlanger, S., « NATO Came Near Kosovo Ground War », International Herald Tribune, 8 novembre 1999, p. 10.

49. Simon, H. A., Models of Bounded Rationality, Cambridge, Mass., MIT Press, 1982 (2 vol.) ; Simon, H. A., Reason in Human Affairs, Stanford, CA, Stanford University Press, 1983. Sur les modèles de Simon appliqués aux phénomènes politiques : Simon, H. A., « Human Nature in Politics : The Dialogue of Psychology with Political Science », American Political Science Review, 79, 1985, pp. 293-304 ; Simon, H. A., « Rationality in Political Behavior », Political Psychology, 16 (1), mars 1995, pp. 45-61. L'autobiographie de Simon est également riche d'enseignements, Simon, H. A., Models of My Life, New York, Basic Books, 1991. Sur l'approche de Simon, Mongin, P., « Modèle rationnel ou modèle économique de la rationalité ? ", Revue économique, 1, janvier 1984, pp. 9-63 ; Haine, J.-Y., « Kennedy, Kroutchev et les missiles de Cuba : choix rationnel et responsabilité individuelle », Cultures et conflits, $n^{\circ} 36$, hiver 1999.

50. Simon, H. A., Administration et processus de décision, Paris, Economica, 1983. 
51. Lindblom, C. E., « The Science of Muddling Through », Public Administration Review, 19, 1959, pp. 79-88 ; Lindblom, C. E., « Still Muddling, Not Yet Through », Public Administration Review, 39 (6), novembre-décembre 1979, pp. 517-528.

52. Fitchett, J., « Kosovo Emerging as « Test Case » for Both Clinton and NATO », International Herald Tribune, 15 septembre 1998, p. 10.

53. Cela ne signifie pas que les forces terrestres ne puissent s'inscrire dans cette logique. Voir notamment, Commandement de la doctrine et de l'enseignement militaire supérieur de l'armée de terre, L'action des forces terrestres au contact des réalités. Une nouvelle approche doctrinale, Paris, Armée de terre, janvier 2000, pp. 55-57.

54. Cohen, E. A., « The Mystique of U.S. Air Power », Foreign Affairs, 73 (1), janvierfévrier 1994, pp. 109-124 ; Eliot A. Cohen, "The Meaning and Future of Air Power », Orbis, printemps 1995, pp. 189-200.

55. Schelling, T. C., Stratégie du conflit, op. cit., pp. 250-246 ; Schelling, T. C., Arms and Influence, op. cit., pp. 99-125.

56. Schelling, T. C., Stratégie du conflit, op. cit., 1986, p. 245.

57. Hassner, P., op.cit., pp. 1224-1233.

58. Hassner, P., op.cit., pp. 1224-1233.

59. Kaufman, W., «Limited Warfare », dans Kaufman, W., et. al., Military Policy and National Security, Princeton, Princeton University Press, 1956 ; Osgood, R. E., Limited War. The Challenge to American Strategy, Chicago, Chicago University Press, 1957 ; Osgood, R. E. \& Tucker, R. W., Force, Order and Justice (1ère éd. 1967), Baltimore, The Johns Hopkins Press, 1971, pp. 3-120 ; Knorr, K., « Is International Coercion Waning or Rising ?», International Security, 1 (4), printemps 1977, pp. 92-110.

\section{INDEX}

Index chronologique : 1999

Mots-clés : diplomatie, guerre, Militaires, Stratégie

Index géographique : Balkans, Kosovo

Thèmes: Pentagone (US Department of Defence) 